\title{
Uncertainty in internet of things: a review
}

\author{
Nur Liesa Mohammad Azemi* and Norfaradilla Wahid \\ Faculty of Computer Science and Information Technology, Universiti Tun Hussein Onn Malaysia, Johor, Malaysia
}

Received: 03-December-2020; Revised: 12-February-2021; Accepted: 18-February-2021

(C2021 Nur Liesa Mohammad Azemi and Norfaradilla Wahid. This is an open access article distributed under the Creative Commons Attribution (CC BY) License, which permits unrestricted use, distribution, and reproduction in any medium, provided the original work is properly cited.

\begin{abstract}
This paper discusses the issue of uncertainty that occurs in the Internet of Things (IoT) environment. The main operation of IoT includes data transmission over sensors and networks via the Internet. There are three types of heterogeneity; namely heterogeneity of data, devices, and networks, which may be the causes that trigger uncertainty in this environment. Based on the study, there are three uncertainty levels that can occur, which are uncertain schema mapping, uncertain data, and uncertain query. As an example, heterogeneous devices which are not compatible with IoT applications will create an opportunity for uncertain data where the data transmitted from the sensor to the application may be partially missing. System failure is likely to happen when these sources of uncertainty generate incorrect inferences and conclusions, thus producing unreliable information. This paper reviews previous researches to find the most efficient approach to handle uncertainty. Discussion is centred on the efficiencies and drawbacks of different uncertainty handling approaches in different domains of knowledge related to IoT while the most relevant of the works are addressed in detail.
\end{abstract}

\section{Keywords}

Heterogeneity, Integration, Internet of things, Uncertainty.

\section{Introduction}

Internet of Things (IoT) refers to a wide range of embedded devices with a unique IP address to enable communication and data sharing over the Internet [1]. In layman's terms, the IoT is a group of physical devices forming a network connected to electronics, software, sensors and network connections that allows data collection and exchange. There exists a massive amount of control and automation without human intervention as the objects are connected and managed with a centralized wireless infrastructure. Thus, the devices can communicate with each other rapidly without the worry of a system failure as it is reduced to a minimum percentage [2]. Besides leading uniformity in daily tasks, IoT also saves time while the applications culminate in better management than humans. Although IoT benefits the world today, there are still challenges to be embraced within the technology. One of them is the issue of uncertainty.

*Author for correspondence

This project is funded by FRGS Grant K060 and UTHM Tier 1 Grant H108.
Uncertainty is a situation that has incomplete or ghost data $[3,4]$ which may lead to severe system analysis.

The uncertainty is mainly generated by the heterogeneity of devices, networks and data collected [5]. Most of the data in IoT are from the sensors, which means that there is a chance of losing the data and eventually cause uncertainty in the IoT environment $[6,7]$.

Previous works [8-14] show that uncertainty issues can exist in different domains with different levels of uncertainty. Based on the research, there are many new and improvised approaches in handling this issue to boost the reliability of the IoT and its environment. Some approaches even work on building new frameworks to handle uncertainty. Hence, this paper is to review efficient approaches to handle uncertainty in an IoT environment based on different characteristics and parameters of multiple domains. The performance evaluation for each approach is also taken into consideration. The criteria of each paper reviewed are discussed in Appendix A. 


\section{Related works}

The increase of IoT applications in smart systems, Industry 4.0, and big data environments have led to research specifically in handling uncertainty in IoT and the related domains [15]. Various approaches have been proposed to cater to different possible issues. The research on uncertainty issues in computing began as early as the 1990s and has continued to expand along with the development of pervasive and ubiquitous computing [16-19]. Unfortunately, not much effort has been done specifically for uncertainties in IoT. However, research has been done in the related area such as in data and web resource management.

$\mathrm{Ba}$ et al. [8] proposed PrXML to integrate websources under uncertainties and dependencies in the maritime domain such as traffic monitoring and tracking of ships. Three platform source examples were given in the paper, and this included web platforms, content-based web editing platforms, and social networking platforms. It was noted in the research that uncertainty can happen while retaining incomplete information on the location of the ships. By implementing the PrXML approach, the result shows that the uncertainty was handled well and the location of the ship is precise. The authors also managed to integrate web sources under uncertainty and dependencies using the proposed approach.

In 2014, a study by Mishra and Ghosh made comparisons between Vague Set and Fuzzy Set based on a similarity measuring method to handle uncertain query issues. The method deduced that the databases that implemented the Vague Set model were more beneficial as compared to the conventional Fuzzy Set model [9].

Diaz et al. [10] tried to solve the issue of uncertainty upon the integration of Cloud Computing in the IoT domain. The authors also focused on security and privacy as cloud storage is vulnerable to threats. The proposed IoT middleware such as LinkSmart, Device Profile for Web Services (DWPS) and LooCI succeeded in integrating Cloud Computing by normalizing, verifying, filtering, compressing and analysing large IoT data amount. However, interoperability between devices and cloud computing is still lacking and may cause more uncertainties to happen.

The work by Magruk [11] studied limiting uncertainties in Smart Building IoT Technology (SBIoT). Data heterogeneity in this environment has caused a high level of uncertainty as it is lacking in interoperability. In order to shift the uncertainty to a lower level, Magruk proposed a cryptographic algorithm approach to overcome the issue in the SBIoT environment. However, the approach only managed to reduce it to a smaller amount.

Assoudi and Lounis [12] focused on coping with uncertainties specifically in schema matching. The approach used is based on the Bayesian Networks and Agent-Based Modeling. From this, a simulation tool, the Schema Matching Agent-Based Simulation (SMAS), was produced. SMAS successfully resolved all the expected schema matching while handling the uncertainty that occurs within the operation. This research, however, resolves a 1:1 matching only and there is room for improvement in solving complex matching.

In an alternate solution towards reducing uncertainty in schema matching, Zhang et al. [13] proposed crowdsourcing with accuracy rates. This led to the formulation of two novel approaches of Correspondence Correctness Question (CCQ) and Multiple-CCQ which adaptively select, publish and manage questions to be asked to the crowd. It can be concluded that they managed to explore the issues of CCQs and the validity of crowd-sourced answers by accuracy rates of the crowds. The drawback in this research is that CCQs' withdrawal or replacement occurs after a waiting time as they are unable to predict the exact amount of answered CCQs.

Uncertain web resources have also become a motivation for Boulaares et al. to model a PrXML approach [14]. The research established an approach for modeling, interpreting and computing the uncertain value under uncertain navigation. The authors also proposed to estimate modeling under several types of uncertain distribution for future work.

Hariri et al. [6] discussed uncertainty in big data analytics. In a massive dataset environment, uncertainty is inherent due to the involvement of a large number of devices and networks. It can be concluded that the possibility of an uncertainty to occur increases when the speed and amount of data also increases. Hence, in mitigating uncertainty, the authors focused on applying different machine learning techniques according to different issues. As an example, incomplete data issues are solved by implementing Fuzzy Logic Theory and deep learning while data scalability is handled by distributed 
learning technique. Based on this approach, four out of the five V's, namely velocity, variety, volume, and veracity, in Big Data uncertainty handling were catered to while data related to value (e.g., data related in decision making in the specific domain) is still lacking in handling the issue of uncertainty.

Various approaches have been used to solve uncertainty from different levels. However, previous researches in this field can only handle a small amount of uncertainty solving as summarized in Table 1. From the table, we can see a few works that are based on a similar approach (e.g. Probabilistic XML). Each of them differs from one another as each tries to solve uncertainty in different domains of the study.

For example, works based on PrXML consist of three major operations, which are modeling, querying and updating. In [8], the work only selected modeling as the operation in handling uncertain web data while implementing the crowd sourcing approach in data integration. The application of PrXML in [14] covers the operation of modeling, query evaluation and updating the query to cater to the uncertainty that occurs in web data resources. Based on [8, 14], it shows that PrXML manages to handle the problem in the different domains of knowledge. Hence, it opens to a new consideration to extend a similar approach to handle uncertainty in the IoT environment.

\section{Uncertainty in the internet of things \\ 3.1Internet of things (IoT)}

IoT is defined as an embedded device with a unique IP address that is able to communicate and share data with each other via the Internet [20]. IoT extends Internet connectivity towards traditional devices such as laptops and smartphones to a manifold range of devices that utilize embedded technology. Atzori et al. [20] verified that the basic idea of implementing pervasive computing towards various things or objects has made life easier.

There is an increase in the application of IoT in multiple domains such as smart systems, healthcare, and business. The rapid growth of mobile apps and wearable devices has made it possible to remotely monitor patient's health via medical devices on an IoT platform [21-24].

However, heterogeneity has become a challenge as countless manufacturers and developers try to establish their own devices and systems. Heterogeneity is taken from the root word 424 heterogeneous which means incommensurable through being of different kinds, degrees, or dimensions. In this instance, heterogeneity may be caused by the various kinds of data, devices, and networks used to develop an IoT system. Thus, it is indeed crucial for IoT applications to address the hindrance of heterogeneity and sanction the exchange of information across platforms and applications [25]. Today, systems implement a wide variety of network connectivity options, proprietary or standardspredicated protocols, and communication methods that are incipient to the IoT. Heterogeneity betokens that there are variations in data, devices or networks [26]. However, these variations can trigger the occurrence of uncertainty in the IoT ecosystem.

Data heterogeneity. Data becomes heterogeneous in the IoT environment as the data collected is multimodel, diverse, voluminous and supplied often in high speed [27]. Although there are recent advances in the IoT framework [28-30], the process of capturing, storing, and processing a huge amount of heterogeneous sensor data has somehow become formidable. The enlargement in the number and variety of sensors has made it strenuous to manage data utilization, determine the kind of services to provide with the data and ascertain the target users [31].

Device heterogeneity. Heterogeneity makes IoT devices hardly interoperable. Therefore, the heterogeneity of devices is a high-priority critical issue which must be resolved [31] by working on the issue of devices with different performances, networking capabilities, widely differing hardware, platform capabilities, usage patterns and remaining battery capacity.

Network heterogeneity. A heterogeneous network is a network connecting computers and other devices with different operating systems and/or protocols. For example, local area networks (LANs) that connect Microsoft Windows and Linux based personal computers with Apple Macintosh computers are heterogeneous $[32,33]$. Along with the development of the IoT, the requirement for services that bridge multiple IoT application domains has increased to realize the efficiency gains promised by the IoT.

\subsection{The uncertainty}

According to Knight [4], uncertainty is defined as a deviation from the expected states that avoid the user from the use of any probability on the resulting establishment for an action or decision. To explain 
uncertainty in IoT, one of the many possible situations can be seen when data reading from sensors becomes unreliable as it is affected by the surroundings such as wind and temperature. In IoT, uncertainty may happen in any aspect from the levels of uncertainty [20].
The Motivating Example. As an example, a user with a wearable device would like to view his daily steps tracking and body monitoring such as blood pressure, heart rate, and body temperature. Therefore, the user

Table 1 Comparative analysis on related works

\begin{tabular}{|c|c|c|c|c|c|}
\hline Author & Issue & $\begin{array}{l}\text { Level of } \\
\text { uncertainty }\end{array}$ & Domain & Approach & Limitations \\
\hline $\begin{array}{l}\text { Ba, et al., } \\
\text { 2014. [8] }\end{array}$ & $\begin{array}{l}\text { To integrate } \\
\text { web sources } \\
\text { under } \\
\text { uncertainties } \\
\text { and } \\
\text { dependencies. }\end{array}$ & Uncertain Data & $\begin{array}{l}\text { Web } \\
\text { Resources }\end{array}$ & $\begin{array}{l}\text { Probabilistic } \\
\text { XML }\end{array}$ & $\begin{array}{l}\text { Imprecise web sources caused by } \\
\text { imperfect human knowledge has to } \\
\text { be taken into consideration to } \\
\text { avoid uncertainty. }\end{array}$ \\
\hline $\begin{array}{l}\text { Mishra and } \\
\text { Ghosh, } \\
\text { 2014. [9] }\end{array}$ & $\begin{array}{l}\text { To compare } \\
\text { between Vague } \\
\text { Set or Fuzzy } \\
\text { Set efficiency } \\
\text { in handling } \\
\text { uncertain query } \\
\text { processing. }\end{array}$ & Uncertain Query & DBMS & $\begin{array}{l}\text { Similarity } \\
\text { Measure }\end{array}$ & $\begin{array}{l}\text { Vague Set model is more fruitful in } \\
\text { processing uncertain queries. }\end{array}$ \\
\hline $\begin{array}{l}\text { Diaz et al., } \\
\text { 2015. [10] }\end{array}$ & $\begin{array}{l}\text { To prevent } \\
\text { uncertainty } \\
\text { upon the } \\
\text { integration of } \\
\text { IoT on the } \\
\text { cloud } \\
\text { computing } \\
\text { platform. }\end{array}$ & Uncertain Data & IoT & $\begin{array}{l}\text { Provide } \text { IoT } \\
\text { middleware }\end{array}$ & $\begin{array}{l}\text { The integration of IoT in cloud } \\
\text { computing platforms is successful } \\
\text { but lacks interoperability between } \\
\text { the device and some of the } \\
\text { proposed middleware. }\end{array}$ \\
\hline $\begin{array}{l}\text { Magruk, } \\
\text { 2015. [11] }\end{array}$ & $\begin{array}{l}\text { To overcome } \\
\text { uncertainty in } \\
\text { Smart Building } \\
\text { IoT technology. }\end{array}$ & Uncertain Data & IoT & $\begin{array}{l}\text { Crypto- } \\
\text { graphic } \\
\text { Algorithm }\end{array}$ & $\begin{array}{l}\text { The algorithm manages to handle } \\
\text { small scale uncertainty in SBIoT. }\end{array}$ \\
\hline $\begin{array}{l}\text { Assoudi and } \\
\text { Lounis, } \\
\text { 2015. [12] }\end{array}$ & $\begin{array}{l}\text { To cope with } \\
\text { uncertainty in } \\
\text { schema } \\
\text { matching. }\end{array}$ & $\begin{array}{l}\text { Uncertain } \\
\text { Schema } \\
\text { Mapping }\end{array}$ & $\begin{array}{l}\text { Business } \\
\text { Information } \\
\text { System }\end{array}$ & $\begin{array}{l}\text { Bayesian } \\
\text { Networks and } \\
\text { Agent-Based } \\
\text { Modeling }\end{array}$ & $\begin{array}{l}\text { The model only resolved } 1: 1 \\
\text { matching of the expected schema } \\
\text { matching but not complex } \\
\text { matching. }\end{array}$ \\
\hline $\begin{array}{l}\text { Zhang et al., } \\
\text { 2018. [13] }\end{array}$ & $\begin{array}{l}\text { To apply crowd } \\
\text { sourcing to } \\
\text { reduce } \\
\text { uncertainty. }\end{array}$ & $\begin{array}{l}\text { Uncertain } \\
\text { Schema } \\
\text { Mapping }\end{array}$ & $\begin{array}{l}\text { Data } \\
\text { Integration }\end{array}$ & $\begin{array}{l}\text { CCQ and } \\
\text { Multiple CCQ }\end{array}$ & $\begin{array}{l}\text { The two novel approaches } \\
\text { managed to reduce uncertainty in } \\
\text { schema matching. However, the } \\
\text { approach is unable to predict the } \\
\text { amount of answered CCQs. }\end{array}$ \\
\hline $\begin{array}{l}\text { Boulaares et } \\
\text { al., } 2018 . \\
{[14]}\end{array}$ & $\begin{array}{l}\text { To model an } \\
\text { approach for } \\
\text { uncertain } \\
\text { representation } \\
\text { and navigation. }\end{array}$ & Uncertain Data & $\begin{array}{l}\text { Web } \\
\text { Resources }\end{array}$ & $\begin{array}{l}\text { Probabilistic } \\
\text { XML }\end{array}$ & $\begin{array}{l}\text { Limited in handling uncertainty for } \\
\text { a single type of uncertain } \\
\text { distribution only. }\end{array}$ \\
\hline $\begin{array}{l}\text { Hariri et al., } \\
\text { 2019. [6] }\end{array}$ & $\begin{array}{l}\text { To evaluate } \\
\text { uncertainty in } \\
\text { big data and } \\
\text { handle it. }\end{array}$ & Uncertain Data & $\begin{array}{l}\text { Big Data } \\
\text { Analytics }\end{array}$ & $\begin{array}{l}\text { Machine } \\
\text { Learning } \\
\text { techniques } \\
\text { mitigation }\end{array}$ & $\begin{array}{l}\text { Focus on handling uncertainty in } \\
\text { four out of the five V's (e.g., } \\
\text { volume, variety, velocity and } \\
\text { veracity) but less work done to } \\
\text { cater uncertainty in value. }\end{array}$ \\
\hline
\end{tabular}


installs an IoT application that can fetch data from his wearable device. There is a possibility of uncertainty during the data transmission process as the device data is accessible by the application. The data displayed in the application may consist of uncertainties such as delayed or missing data caused by Internet disconnection and inconsistent data. Tags are readable by various readers at the same time, thus, it is possible to get inconsistent data on the exact location of tags. Figure 1 shows the example where a data resource Wearable A links to Application A with the possibility of uncertainty to occur during data transmission.

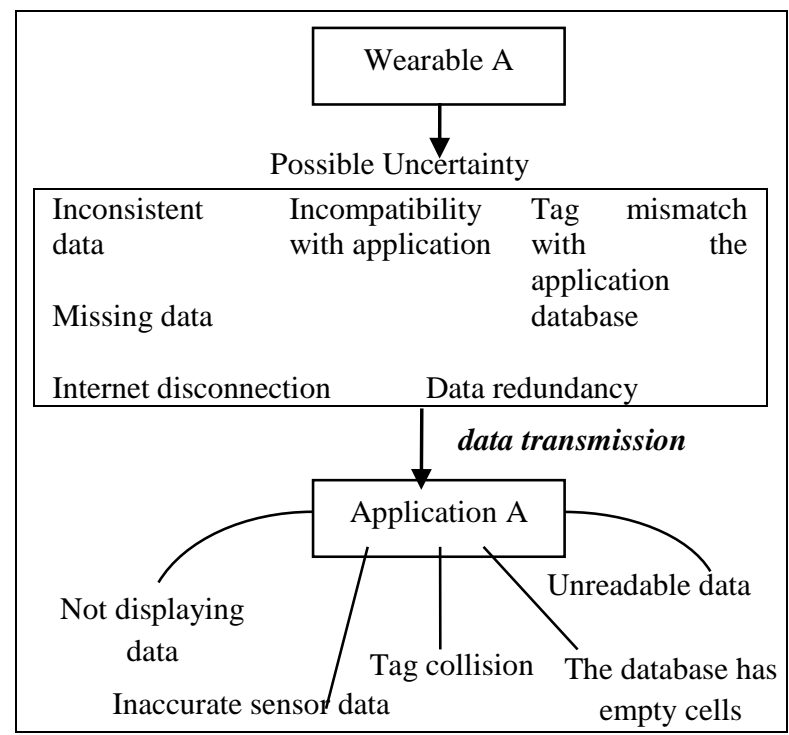

Figure 1 Possibility of uncertainty

\subsection{Level of uncertainty}

There are three levels of uncertainty, which are uncertain schema mapping, uncertain data and uncertain queries [5]. This section will briefly discuss each mentioned level.

3.3.1Uncertain schema mapping

Schema mapping refers to finding correspondences between the elements of two given schemata. In an uncertain schema mapping, inaccuracy may persist due to the imprecise pairing of attributes between the source schema and target schema $[5,13]$. Uncertainty is possible to happen if the attributes differ. Hence, the attributes must be given a standardized format to prevent uncertainties from happening.

Example 1 Consider a data source $\mathrm{S}$, which describes a sensor from Wearable A (refer to Figure 1) by its type, IP address, temperature readings in Celsius $\left({ }^{\circ} \mathrm{C}\right)$ and temperature readings in Fahrenheit $(F)$, and the target schema $\mathrm{T}$, which describes another sensor by its name, ID and temperature reading:

$S=($ code, IPaddress, tempC, tempF $)$

$T=$ (name, ID, temp)

Ambiguity exists when the schema mapping tools produce a set of possible mapping. This mapping is required to define the various views and data representations in an IoT database. In this example, possible mappings from the schema are generated.

$m_{1}=\{($ code, name $),($ IPaddress, ID),$($ tempC, temp $)\}$

$m_{2}=\{($ code, name $),($ IPaddress, ID $),($ tempF, temp $)\}$

\subsubsection{Uncertain data}

In an uncertain data level, data is often extracted from unreliable unstructured or semi-structured sources [34], such as devices that generate sensor reading data. Uncertain data are inherent in major applications, such as environmental surveillance, medication and smart systems [35]. Additionally, data veracity is another term that is usually related to data uncertainties, especially in any big data management. It refers to the data noise, bias, and abnormality that may lead to a more significant problem. For example, uncertain data produces a particular diagnosis that fits a patient's symptoms. If treatment is given to the patient based on that diagnosis, it may lead to a fatality.

In some cases, uncertain data is caused by imperfect human knowledge [8]. Humans, or specifically social network users, may spread imprecise information on different platforms; thereby causing uncertainty. Given a scenario where a temperature sensor measures room temperature in a smart room control application, the temperature reading might be imprecise due to room humidity or wind flowing into the room from open windows. These factors will affect the sensor's reading, thus producing uncertain data.

3.3.3Uncertain queries

The final level of uncertainty is uncertain queries, in which the system is required to translate the queries into a structured form. Only a few researches have been done to determine uncertain queries. According to the study conducted by Mishra and Ghosh [9], uncertain queries are preferably done in the relational database by the Vague Set model. An uncertain query means that there can be a possible query that has no executable plan. As can be seen in Table 2, a temperature may consist of two different readings (e.g. in Celsius or Fahrenheit). The following applies supposing that the system receives a query $Q$ 
formulated using the target schema and asks for temperature reading:

$Q=$ SELECT temp FROM T

Using the possible mapping, $Q$ can be reformulated into two uncertain queries that lack the information on which temperature unit should it generate:

$Q_{1}=$ SELECT tempC FROM S

$Q_{2}=$ SELECT tempF FROM S

\section{Uncertainty in IoT integration}

There are increased possibilities of uncertainty in the IoT environment as indicated by the viability of IoT technology. Previous works done in [34-39] discussed the integration of IoT with another domain. Based on these works, it can be deduced that uncertainty can occur upon integration and under certain circumstances.

Islam et al. [34] focused their work in the field of IoT-based healthcare. The integration between IoT and healthcare has continued to arise in the implementation of IoT sensors and devices on healthcare equipment, accommodation, and even medicine. Sensors used in healthcare like blood pressure monitoring sensors are usually designed as a wearable device. Wireless Body Area Network (WBAN) connects these devices via the Internet through an appropriate gateway. However, if the gateway faces failure, the device will produce disruptive unreliable data and there will be a room of uncertainty. Different devices and networks contain different settings; hence, uncertainty will take place in this diverse range since there is no standardization of protocols and rules to interact with each other. Besides that, IoT-based healthcare devices require an update in every versioning due to the limitations of a few categories of diseases only. Imprecise disease detection will somehow lead to a larger uncertainty that needs to be solved.

Nowadays, there is rapid evolution in wireless communication devices. From smartphones to smart watches, people find it necessary to have each of them connected to the Internet just to make their daily tasks easier. The integration between the Internet of Things and devices was studied by Elkhodr et al. [36], which stated that uncertainty is majorly caused by heterogeneous devices manufactured with different capabilities, hardware and embedded sensors. This has caused imprecise sensor readings that are transmitted through integration. The authors concluded that IoT requires the standards as mentioned in [34] to enable horizontal platforms with interoperability across a mixture of devices that are unpredictable, fragmented and lacks interaction among each other.

Besides the research done in IoT-based healthcare and IoT-based devices, the integration between IoT into cloud computing is also an interesting topic as both are two different technologies. The merging of both parties acts as an enabler of a vast number of application scenarios such as in a smart city, environmental monitoring, and smart transportation. CloudIoT paradigm introduced by Botta et al. [37] in 2014 has become a baseline to two other related works by Atlam et al. [38] and Stergiou et al. [39] that focused on IoT-based cloud. In this unification, IoT benefits virtually unlimited capabilities and cloud resources are compensated in its technological aspects such as storage and processing while cloud computing is able to expand the characteristics of IoT that deals with real-world data in a distributed and more dynamic manner. Thus, producing new models such as Sensor as a Service (SenaaS), Data as a Service (DaaS), and Sensor and Automation as a Service (SAaaS) [37].

In [38], the authors highlighted that mitigating specific IoT application data into the cloud may lead to complexity related to heterogeneous issues from the data, devices, and networks. Huge and streaming sensor data that are timely processed are a common factor that causes uncertainty and network constraints. Besides that, the problem of missing data samples from the integration of both IoT and cloud is a crucial limitation towards providing meaningful data in IoT-based cloud applications.

A few functionalities were achieved through the integration of IoT and cloud in [39], such as the usage of IoT-based cloud in sensor connection and sensor readings that are accessible via the cloud. However, there is still the possibility of uncertainty irrespective of how cloud and IoT complement each other. As taken in the running example from this paper, in the context of smart transportation, networking and communication have become uncertain as vehicles are always moving and precise information is not obtained.

The integration within different IoT application domains such as smart energy and smart home, smart education and smart building, and smart transportation and smart city, with different data types and terms used in each domain are stated as possible to happen by Alavi et al. [40]. The key characteristic is to find almost similar keywords 
between two domains to undergo schema mapping and allow the integration to happen. As an example, in the healthcare domain, a patient with a walking disability is using a smart wheelchair embedded with a tracking sensor. Data from the sensor is used to track street information and update traffic information provided by the smart city domain.
However, there is still a gap in the research to solve uncertainty within the IoT environment even with the number of contributions from the aforementioned works. A comparative summary between every research reviewed is shown in Table 2.

Table 2 Comparative study of uncertainty in IoT integration

\begin{tabular}{|c|c|c|}
\hline Author & IoT-based in domain & Uncertainty issue \\
\hline Islam et al., 2015 [34] & Healthcare & $\begin{array}{l}\text { - Sensor detection is limited to a few categories of } \\
\text { diseases only, uncertain schema mapping occurs } \\
\text { when new terms in medicine are published. } \\
\text { A diverse range of products and devices cause } \\
\text { uncertain data transmission among } \\
\text { heterogeneous devices. }\end{array}$ \\
\hline Elkhodr et al., 2016 [36] & Devices & $\begin{array}{l}\text { - The heterogeneous device leads to unreliable and } \\
\text { imprecise data reading sent over the IoT } \\
\text { applications. }\end{array}$ \\
\hline Atlam et al., 2017 [38] & Cloud Computing & $\begin{array}{l}\text { - The timely processing of large and real-time } \\
\text { sensor data cause delays in sensor networks. } \\
\text { - Missing data samples during integration. }\end{array}$ \\
\hline Stergiou et al., 2018 [39] & Cloud Computing & $\begin{array}{l}\text { - The mobility of a sensor device will cause } \\
\text { unreliable and imprecise data. }\end{array}$ \\
\hline
\end{tabular}

\section{Conclusion and future work}

This paper has discussed the issues of uncertainty in every level from uncertain schema mapping to uncertain data to uncertain queries in the Internet of Things. The existence of IoT enables things to be connected endlessly from a wearable device to a smart home to healthcare. IoT emboldens the communication between devices, famously known as Machine-to-Machine (M2M) communication [41], [42]. Every incipient technology faces manifold challenges, and one of them is uncertainty. Works in [5-14] and [34-39] show that issues of uncertainty can exist in different domains with different levels of uncertainty. There is also the possibility of having uncertainty in the integration between IoT and other domains. Therefore, an efficient approach to handle uncertainty in IoT integration is crucial to ensure flawless operations in IoT applications.
This paper has reviewed different techniques in mitigating uncertainty in multiple domains, where various approaches have been proposed to solve different related issues. As can be seen in Section 2, some of the works utilized the same strategy in handling uncertainties in the different domains of knowledge. One of the potential approaches is Probabilistic XML which can be expanded to minimize the amount of uncertain schema mapping, data, and queries in the IoT ecosystem. Security issues, middleware malfunctions or data veracity must be prevented from creating any chance of uncertainty. Hence, there is room for improvement in order to maintain the performance of an IoT application that can help in precise decision making. Due to the evolving demands and trends nowadays, additional studies must be carried out on the uncertainty in different environments, e.g., web of things and semantic web of things.

\section{Appendix}

The complete criteria, details and sources of each paper reviewed can be found from Table 3, Figure 2 and Figure 3.

Table 3 Papers collection criteria

\begin{tabular}{lllll}
\hline Reference & Year & Source & \multicolumn{2}{c}{ Criteria } \\
\cline { 4 - 5 } & & & Inclusion & Exclusion \\
\hline Ba et al. [8] & 2014 & Springer & Apply Probabilistic XML approach & Web resources domain, use offline data \\
\hline $\begin{array}{l}\text { Mishra and } \\
\text { Gosh [9] }\end{array}$ & 2014 & IJCCC & Handle uncertain query & Offline data \\
\hline $\begin{array}{l}\text { Diaz et al } \\
{[10]}\end{array}$ & 2015 & Elsevier & Cater uncertainty in IoT domain & Focus more on security attack \\
\hline 428 & & & & \\
& & & &
\end{tabular}


International Journal of Advanced Technology and Engineering Exploration, Vol 8(75)

\begin{tabular}{lllll}
\hline Reference & Year & Source & \multicolumn{2}{c}{ Criteria } \\
\cline { 3 - 5 } & & & Inclusion & Exclusion \\
\hline Magruk [11] & 2015 & Elsevier & Cater uncertain data in IoT domain & Focus on smart building only \\
\hline $\begin{array}{l}\text { Assoudi and } \\
\text { Lounis [12] }\end{array}$ & 2015 & Springer & Use BNN method & Solve simple schema matching only \\
\hline $\begin{array}{l}\text { Zhang et al. } \\
\text { [13] }\end{array}$ & 2018 & IEEE & Reduce uncertainty in schema mapping & $\begin{array}{l}\text { Crowd sourcing method is not suitable to } \\
\text { use in IoT }\end{array}$ \\
\hline $\begin{array}{l}\text { Boulaares et } \\
\text { al. [14] }\end{array}$ & 2018 & IEEE & Apply Probabilistic XML approach & Offline data \\
\hline $\begin{array}{l}\text { Islam et al. } \\
\text { [34] }\end{array}$ & 2015 & IEEE & Healthcare device heterogeneity & Focus on device and security \\
\hline $\begin{array}{l}\text { Elkhodr et al. } \\
\text { [36] }\end{array}$ & 2016 & arXiv & Device heterogeneity & Focus more on security than uncertainty \\
\hline $\begin{array}{l}\text { Atlam et al. } \\
\text { [38] }\end{array}$ & 2017 & IEEE & Handle uncertainty in Cloud Computing & Different parameters from IoT \\
\hline $\begin{array}{l}\text { Stergiou et al. } \\
{[39]}\end{array}$ & 2018 & Elsevier & Device heterogeneity in Cloud Computing & Focus on security only \\
\hline $\begin{array}{l}\text { Hariri [6] } \\
2019\end{array}$ & Springer & $\begin{array}{l}\text { Handle uncertainty in big data analytics } \\
\text { which is similar to IoT domain }\end{array}$ & $\begin{array}{l}\text { Only cater uncertainty handling in four } \\
\text { out of five V's in big data. }\end{array}$ \\
\hline
\end{tabular}

\section{Sources of Paper}

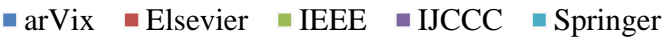

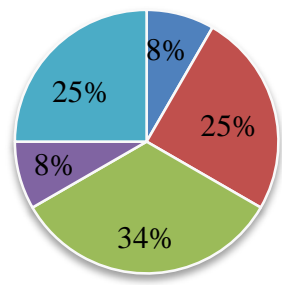

Figure 2 Sources of paper collection

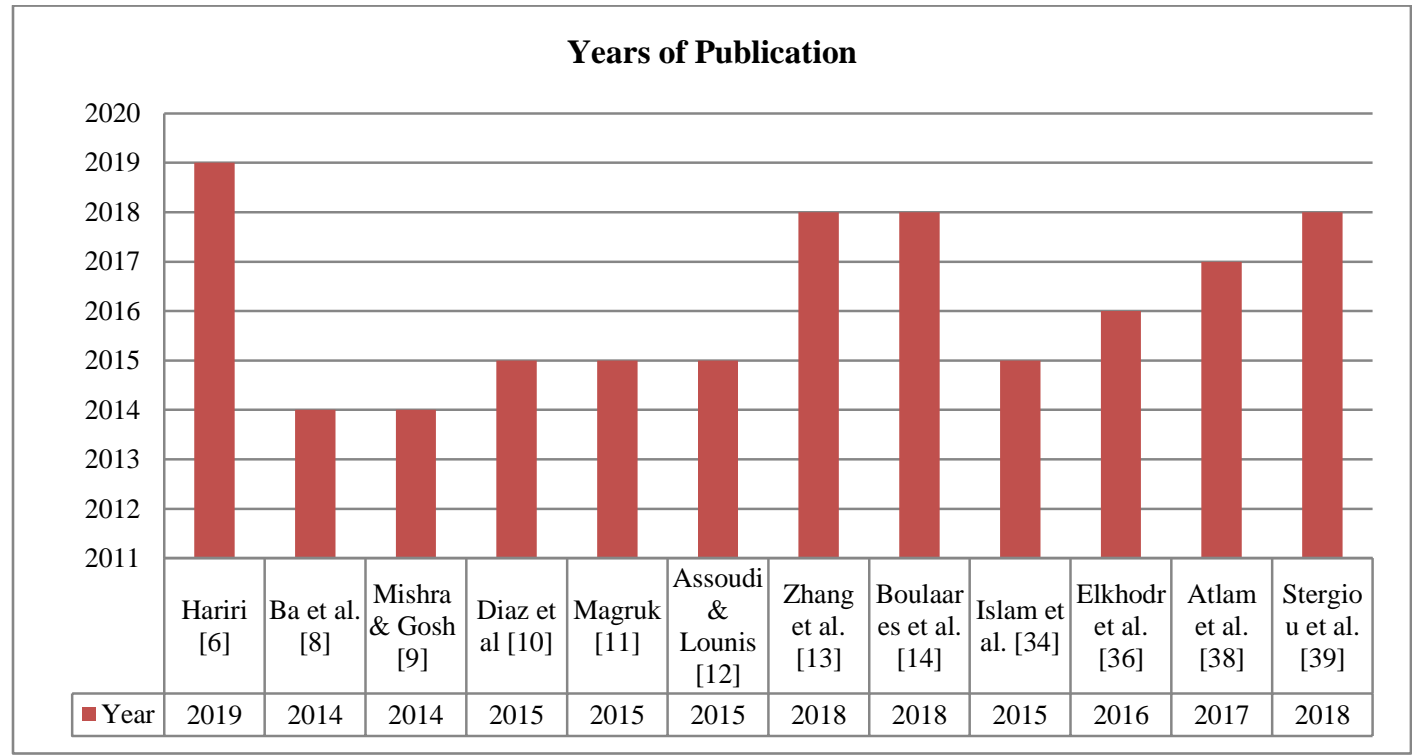

Figure 3 Years of paper publication

429 


\section{Acknowledgment}

The authors would like to thank the Ministry of Education Malaysia for supporting this research under Fundamental Research Grant Scheme Vot No. FRGS/1/2018/ICT04/UTHM/03/1 and partially sponsored by Tier 1 Grant H108 Universiti Tun Hussein Onn Malaysia.

\section{Conflicts of interest}

The authors have no conflicts of interest to declare.

\section{References}

[1] Derrien S, Meye P, Raipin P. Thing in, a research platform for the Web of Things. In 27th international symposium on modeling, analysis, and simulation of computer and telecommunication systems 2019 (pp. 431-2). IEEE.

[2] Gyrard A, Bonnet C, Boudaoud K, Serrano M. Assisting iot projects and developers in designing interoperable semantic web of things applications. In international conference on data science and data intensive systems 2015 (pp. 659-66). IEEE.

[3] Zhao W, Jiang H, Tang K, Pei W, Wu Y, Qayoom A. Knotted-line: a visual explorer for uncertainty in transportation system. Journal of Computer Languages. 2019; 53:1-8.

[4] Knight FH. Risk, uncertainty and profit. Houghton Mifflin; 1921.

[5] Gal A, Roitman H, Shraga R. Learning to rerank schema matches. IEEE Transactions on Knowledge and Data Engineering. 2019:1-14.

[6] Hariri RH, Fredericks EM, Bowers KM. Uncertainty in big data analytics: survey, opportunities, and challenges. Journal of Big Data. 2019; 6(1):1-6.

[7] Alagar V, Wan K. Understanding and measuring risk due to uncertainties in IoT. In international conference on smart internet of things 2019 (pp. 484-8). IEEE.

[8] Ba ML, Montenez S, Tang R, Abdessalem T. Integration of web sources under uncertainty and dependencies using probabilistic XML. In international conference on database systems for advanced applications 2014 (pp. 360-75). Springer, Berlin, Heidelberg.

[9] Mishra J, Ghosh S. Uncertain query processing using vague set or fuzzy set: which one is better? International Journal of Computers Communications \& Control. 2014; 9(6):730-40.

[10] Díaz M, Martín C, Rubio B. State-of-the-art, challenges, and open issues in the integration of Internet of things and cloud computing. Journal of Network and Computer Applications. 2016; 67:99117.

[11] Magruk A. The most important aspects of uncertainty in the internet of things field-context of smart buildings. Procedia Engineering. 2015; 122:220-7.

[12] Assoudi H, Lounis H. Coping with uncertainty in schema matching: Bayesian networks and agent-based modeling approach. In international conference on etechnologies 2015 (pp. 53-67). Springer, Cham.
[13] Zhang CJ, Chen L, Jagadish HV, Zhang M, Tong Y. Reducing uncertainty of schema matching via crowdsourcing with accuracy rates. IEEE Transactions on Knowledge and Data Engineering. 2018; 32(1):135-51.

[14] Boulaares S, Omri A, Sassi S, Benslimane D. A probabilistic approach: a model for the uncertain representation and navigation of uncertain web resources. In international conference on signal-image technology \& internet-based systems 2018 (pp. 2431). IEEE.

[15] Magruk A. Uncertainty in the sphere of the industry 4.0-potential areas to research. Business, Management and Education. 2016; 14(2):275-91.

[16] Ning C, You F. Optimization under uncertainty in the era of big data and deep learning: When machine learning meets mathematical programming. Computers \& Chemical Engineering. 2019; 125:43448.

[17] Tchernykh A, Schwiegelsohn U, Talbi EG, Babenko M. Towards understanding uncertainty in cloud computing with risks of confidentiality, integrity, and availability. Journal of Computational Science. 2019; 36:1-9.

[18] Kale SS, Patil PS. Data mining technology with fuzzy logic, neural networks and machine learning for agriculture. In data management, analytics and innovation 2019 (pp. 79-87). Springer, Singapore.

[19] Zhou Z, Liao H, Zhao X, Ai B, Guizani M. Reliable task offloading for vehicular fog computing under information asymmetry and information uncertainty. IEEE Transactions on Vehicular Technology. 2019; 68(9):8322-35.

[20] Atzori L, Iera A, Morabito G. The internet of things: a survey. Computer Networks. 2010; 54(15):2787-805.

[21] Zhang X, Deng Z, Parvinzamir F, Dong F. MyHealthAvatar lifestyle management support for cancer patients. Ecancermedicalscience. 2018; 12:117.

[22] Arfaoui A, Kribeche A, Senouci SM. Context-aware anonymous authentication protocols in the internet of things dedicated to e-health applications. Computer Networks. 2019; 159:23-36.

[23] Bellagente P, Depari A, Ferrari P, Flammini A, Sisinni E, Rinaldi S. $\mathrm{M}^{3}$ IoT-message-oriented middleware for M-health internet of things: design and validation. In international instrumentation and measurement technology conference 2018 (pp. 1-6). IEEE.

[24] Qi J, Yang P, Min G, Amft O, Dong F, Xu L. Advanced internet of things for personalised healthcare systems: a survey. Pervasive and Mobile Computing. 2017; 41:132-49.

[25] Hamidouche L, Sens P, Monnet S, Refauvelet D. Toward heterogeneity-aware device-to-device data dissemination over Wi-Fi networks. In international conference on parallel and distributed systems 2017 (pp. 105-12). IEEE

[26] Jabbar S, Ullah F, Khalid S, Khan M, Han K. Semantic interoperability in heterogeneous IoT infrastructure for healthcare. Wireless 
Communications and Mobile Computing. 2017; 2017:1-11.

[27] Piasco N, Sidibé D, Demonceaux C, Gouet-Brunet V. A survey on visual-based localization: On the benefit of heterogeneous data. Pattern Recognition. 2018; 74:90-109.

[28] Fortino G, Savaglio C, Palau CE, De Puga JS, Ganzha M, Paprzycki $M$, et al. Towards multi-layer interoperability of heterogeneous IoT platforms: The INTER-IoT approach. In integration, interconnection, and interoperability of IoT systems 2018 (pp. 199232). Springer, Cham.

[29] Li S, Da Xu L, Zhao S. 5G Internet of things: a survey. Journal of Industrial Information Integration. 2018; 10:1-9.

[30] Yaqoob I, Hashem IA, Ahmed A, Kazmi SA, Hong CS. Internet of things forensics: Recent advances, taxonomy, requirements, and open challenges. Future Generation Computer Systems. 2019; 92:265-75.

[31] Kazmi A, Jan Z, Zappa A, Serrano M. Overcoming the heterogeneity in the internet of things for smart cities. In international workshop on interoperability and open-source solutions 2016 (pp. 20-35). Springer, Cham.

[32] Fang Z, Zhang F, Zhang D. Fine-grained travel time sensing in heterogeneous mobile networks. In proceedings of the 17 th conference on embedded networked sensor systems 2019 (pp. 420-1).

[33] Delphinanto A, Koonen T, Den Hartog F. End-to-end available bandwidth probing in heterogeneous IP home networks. In consumer communications and networking conference 2011 (pp. 431-5). IEEE.

[34] Islam SR, Kwak D, Kabir MH, Hossain M, Kwak KS. The internet of things for health care: a comprehensive survey. IEEE Access. 2015; 3:678-708.

[35] Zhang K, Wang J, Wang M, Han X. Probabilistic skyline computation on vertically distributed uncertain data. In 39th international conference on distributed computing systems (ICDCS) 2019 (pp. 154-63). IEEE.

[36] Elkhodr M, Shahrestani S, Cheung H. The internet of things: new interoperability, management and security challenges. arXiv preprint arXiv:1604.04824. 2016.

[37] Botta A, De Donato W, Persico V, Pescapé A. Integration of cloud computing and internet of things: a survey. Future Generation Computer Systems. 2016; 56:684-700.
[38] Atlam HF, Alenezi A, Alharthi A, Walters RJ, Wills GB. Integration of cloud computing with internet of things: challenges and open issues. In international conference on internet of things (iThings) and IEEE green computing and communications (GreenCom) and IEEE cyber, physical and social computing (CPSCom) and IEEE smart data (SmartData) 2017 (pp. 670-5). IEEE.

[39] Stergiou C, Psannis KE, Kim BG, Gupta B. Secure integration of IoT and cloud computing. Future Generation Computer Systems. 2018; 78:964-75.

[40] Alavi AH, Jiao P, Buttlar WG, Lajnef N. Internet of things-enabled smart cities: state-of-the-art and future trends. Measurement. 2018; 129:589-606.

[41] Zhou Z, Guo Y, He Y, Zhao X, Bazzi WM. Access control and resource allocation for M2M communications in industrial automation. IEEE Transactions on Industrial Informatics. 2019; 15(5):3093-103.

[42] Lv T, Ma Y, Zeng J, Mathiopoulos PT. Millimeterwave NOMA transmission in cellular M2M communications for internet of things. IEEE Internet of Things Journal. 2018; 5(3):1989-2000.

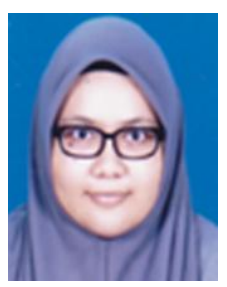

Nur Liesa Mohammad Azemi is a Master's Degree student in Information System at Universiti Tun Hussein Onn Malaysia. She received her Bachelor's Degree in Computer Science (Web Technology) from the same university. Her research focuses on the Internet of Things.

Email: theliesazemi@gmail.com

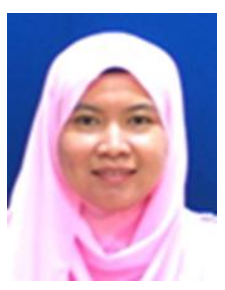

Dr. Norfaradilla Wahid is a Lecturer of Faculty of Computer Science and Information Technology at Universiti Tun Hussein Onn Malaysia. She received her B.Sc (Comp. Sc.) with Honors and M. Sc (Comp. Sc.) from Universiti Teknologi Malaysia in 2005 and 2008 respectively. She continued her PhD study at La Trobe University, Australia where she obtained her $\mathrm{PhD}$ in XML Constraint Preservation. Dr Norfaradilla Wahid current interest involve in Internet of Thing Semantic Data Management.

Email: faradila@uthm.edu.my 